\title{
Simulating the dynamics in two-settlement electricity markets via an agent-based approach
}

\author{
Daniel J. Veit ${ }^{1}$, Anke Weidlich ${ }^{1}$, Jian Yao $^{2}$, Shmuel S.Oren ${ }^{2}$ * \\ 1 Chair for Business Administration and Information Systems - E-Business \\ and E-Government, University of Mannheim, Germany \\ 2 Department of Industrial Engineering and Operations Research, University \\ of California, Berkeley, CA 94720
}

(Received July 10 2006, Accepted October 15 2006)

\begin{abstract}
This paper studies the dynamics in two-settlement electricity markets. In these markets, energy producers sign strategic forward contracts in the forward market, and engage in spatial oligopolistic competition in the spot market. We develop an agent-based model for simulating the outcomes of such markets. Numerical simulations imply that the access to the forward market leads to more competitive behaviors of the suppliers in the spot market, and thus to lower spot energy prices.
\end{abstract}

Keywords: two settlements, electricity markets, Cournot, agent-based simulation

\section{Introduction}

In the last two decades, electricity reform has been prevailing in many countries around the world. In designing competitive markets, many electricity sectors have implemented or are about to implement multiple settlements. A multi-settlement approach consists of at least a forward market and a spot (real-time) market, each producing its own financial settlements. Multiple settlements mean that charges for demand and payments to supply occur in both the forward and spot market. The forward market schedules the production and consumption before the operating day, where as the real-time market reconciles any differences between the schedule in the forward market and the real-time load while observing security constraints and flow limits on transmission lines.

Generation and demand bids that clear the forward market are settled at forward prices. These bids are applied to each operating period of the spot market and to each location in the network. (In addition, incremental offers and decremental bids can be submitted, which indicates the prices at which supply or demand are willing to increase or decrease their injections or withdrawals in the system. These "incs" and "decs" are the tools for market participants to adjust their positions in the forward market.) The settlements in the forward market can be either financially or physically binding. At the spot, scheduled suppliers must produce the committed quantity or, otherwise, buy power to balance their positions. Likewise, wholesale buyers of electricity pay for and lock in their rights to consume the quantities cleared at the forward prices.

While the forward market settles the schedule and financial terms of energy production and consumption on the operating day, the quantities that clear the forward market do not necessarily match the real-time load. For generators, the spot market provides additional opportunities of offering energy into the market. Megawatts over- or under-produced relative to the forward commitments are settled at real-time prices.

Multiple settlements also allow market participants to secure stable prices and to reduce their vulnerability to price fluctuations in the spot market. Price volatility stems from unforeseen events, such as demand

\footnotetext{
*E-mail address: veit, weidlich@uni-mannheim.de; jyao, oren@ieor.berkeley.edu.
} 
uncertainty and transmission and generation outages. Because the forward market is not significantly affected by these events, it produces more stable prices than the spot market. Therefore, forward contracts can help market participants hedge spot price risks. Moreover, because forward prices influence real-time behaviors, they generate more stable profit for generators and incite wholesale consumers to better manage their consumption patterns. Finally, expanding the horizon for energy transactions to multiple settlements makes the market more liquid, more competitive and more economically efficient. Thus, opportunities and incentives for market participants to manipulate the markets are limited.

Research on two-settlement markets has been conducted in (Allaz 1992) ${ }^{[1]}$, (Allaz and Vila 1993) $)^{[2]}$, (Kamat and Oren 2004) ${ }^{[9]}$, (Yao, Oren and Adler 2005a) ${ }^{[13]}$, (Yao, Oren and Adler 2005b) ${ }^{[14]}$ and (Yao, Adler and Oren 2005c) ${ }^{[12]}$ via analytical and computational approaches. It was shown in these studies that, with two settlements, producers have incentives to enter into forward contracts, which reduce spot market prices and increase social welfare. In this paper, we continue the study on two-settlement markets. In particular, we will develop an agent-based model for simulating market outcomes, and quantify producers' incentives for forward contracts and the impact of such incentives, if any, on spot market prices. While this paper focuses primarily on replicating previous results with the agent-based methodology, the agent approach is potentially more flexible in dealing with richer formulations, accounting for dynamic aspects, multiple settlements (e.g., forward, day-ahead and real-time) and other realistic complexities which had to be abstracted in the analytic and computational equilibrium approaches to the problem.

The remainder of this paper is organized as follows. The next section reviews literature on two-settlement electricity markets and agent-based simulation. In section 3, we present the dynamics of the two-settlement system from a mathematical point of view. The agent-based model is constructed in section 4. Section 5 performs simulation on a stylized version of the Belgian electricity market and interprets the results.

\section{Literature review}

Allaz (1992) ${ }^{[1]}$ shows that, in a single-node system, forward contracting creates a prisoners' dilemma type of game among the producers. He shows that the introduction of a forward market leads the producers to behave more competitively in the spot market, which increases social welfare and reduces spot prices. Allaz and Vila (1993) $)^{[2]}$ extend this analysis to the case of multiple periods for forward trading, and show stronger effects of the prisoners' dilemma game.

Recent advances in studying two-settlement markets with more realistic settings have been following two frameworks. The first framework is based on numerical methods. Kamat and Oren (2004) ${ }^{[9]}$ analyze twosettlement markets over 2- or 3-node networks, and extend Allaz' results to a system with uncertain transmission capacities in the spot market. Recent work further extends the above results to more realistic multi-node and multi-zone networks. Yao, Oren and Adler (2005a) ${ }^{[13]}$ consider flow constraints, system contingencies and demand uncertainties in the spot market. Their numerical tests show that, like in the simple cases, generation firms have the incentive to engage in forward contracting, which increases social surplus and reduces spot prices. In their subsequent work, Yao, Oren and Adler (2005b) ${ }^{[14]}$ consider two alternative mechanisms for capping prices. They observe that a forward cap, which may be induced by free entry of new generation capacity, increases firms' incentives for forward contracting, whereas a regulated spot price cap reduces such incentives.

Another stream of research employs agent-based simulation methods for the analysis of electricity markets, e.g. (Bower and Bunn 2000) ${ }^{[3]}$. In these models, the behavior of market participants is implemented into software agents that participate in the markets and act individually to maximize their profits. To our knowledge agent-based models developed until now mostly focus on strategic behavior of electricity market actors in single settlement scenarios. However, an agent-based approach can potentially handle the complexity of multiple settlements. The analysis of a multi settlement scenario in electricity markets is subject of our research presented in this paper.

In one of the first approaches in the agent-based methodology, Bower and Bunn (2000) ${ }^{[3]}$ simulate the England and Wales electricity market. Their focus is the investigation of the impact of different market designs on the allocation outcome. In one case, they compare different bidding time scales, i.e. daily bids versus hourly 
bids. In another case, they examine the effect of a uniform price versus a discriminatory settlement. Agents are endowed with a simple reinforcement learning algorithm which is driven by the goal to maximize profits and to reach a given utilization rate of a power plant. The results of their simulations show that discriminatory settlement leads to higher prices than uniform price settlement, and hourly bidding causes higher prices than daily bidding, due to the fact that the inelastic demand helps the generation companies to reach high market prices in peak demand periods (see also (Bower and Bunn 2001) ${ }^{[4]}$ ).

Czernohous et al. (2003) $)^{[7]}$ use an agent-based approach to derive management decision support in the German electricity market. They model demand and supply agents as individually acting parties and show the feasibility of a combined approach, in which generating companies optimize their offers based on a linear program. In the same model, these offers are submitted into an agent-based market scenario where the demand agents are equipped with different learning strategies.

Nicolaisen, Petrov and Tesfatsion (2001) ${ }^{[11]}$ further develop a reinforcement learning algorithm which is based on (Erev and Roth 1998) ${ }^{[8]}$. This algorithm integrates the effect of experimentation and forgetting by adaptive market participants. The developed model is applied to analyze the individual effects of market structure and agent learning on market power and efficiency outcomes for a computational wholesale electricity market with discriminatory pricing. While the authors' model is highly stylized, it shows that the chosen reinforcement learning algorithm can model agent learning in electricity markets in a satisfactory manner in further agent-based studies, as well as in the research presented in this paper, thus use the same learning representation as given in (Nicolaisen, Petrov and Tesfatsion 2001) ${ }^{[11]}$.

\section{Mathematical presentation of the dynamics}

A two-settlement system comprises two markets: a forward market and a spot market. In the forward market, firms simultaneously enter into forward contracts expecting the strategic effect of their forward contracts on the spot market outcomes. The spot market consists of two stages. In stage one, Nature picks a state defined by a realization of demand uncertainty and system contingencies. In stage two, the firms, whose information sets include the state of nature and all forward commitments, compete in a spatial Nash-Cournot manner while the Independent System Operator (ISO) redispatches generator outputs. In this section, we introduce the market participants' decision problems in a backward order following the formulation in (Yao, Adler and Oren 2005) ${ }^{[12]}$.

\subsection{The spot market}

We consider a centrally-dispatched wholesale spot market. In this market, the ISO controls the transmission system and redispatches generator outputs so as to maximize social welfare while meeting all the network and security constraints. The ISO also sets locational energy prices and transmission charges for bilateral energy transactions. This spot market is subject to demand uncertainty, flow constraints and system contingencies.

The network underlying the spot market is composed of a set $N$ of nodes (or buses), and a set $L$ of transmission lines. There are a set $G$ of generation firms competing in the market, each operating the units at a subset of locations $N_{g} \subseteq N$. For simplicity, we assume that at most one generation firm operates at a node and introduce artificial nodes, if necessary, to meet this assumption.

\subsubsection{The ISO's decision making}

In each state $c \in C$, the ISO determines the import/export $\left\{r_{i}^{c}\right\}_{i \in N}$ at the nodes (using the convention that positive quantities represent imports). These quantities must satisfy the network feasibility constraints, that is, the resulting power flows should not exceed the thermal limits $K_{l}^{c}$ of the corresponding transmission lines in both directions:

$$
-K_{l}^{c} \leq \sum_{i \in N} D_{l, i}^{c} r_{i}^{c} \leq K_{l}^{c}, \quad l \in L
$$


Here, we model the transmission network as a lossless DC approximation of Kirchhoff's laws (see (Chao and Peck 1996 ${ }^{[5]}$ ). Specifically, flows on lines are calculated via power transfer distribution factors $D_{l, i}$ which specify the proportion of flow on a line $l \in L$ resulting from an injection of one-unit electricity at a node $i \in N$ and a corresponding one-unit withdrawal at some fixed reference bus (also known as the slack bus).

Moreover, because electricity is not economically storable, the load and generation must be balanced at all moments. This leads to the energy balancing constraint:

$$
\sum_{i \in N} r_{i}^{c}=0
$$

The ISO aims to maximize the social welfare of the entire system, which is given by the total consumer willingness-to-pay (the aggregated area under the nodal inverse demand curves) minus the total generation cost. Mathematically, it solves the following problem parametric on the firms' production decisions $\left(\left\{q_{i}\right\}_{i \in N}\right)$ :

$$
\begin{array}{ll}
\max _{\left\{r_{i}^{c}\right\}_{i \in N}} & \sum_{i \in N}\left(\int_{0}^{r_{i}^{c}+q_{i}^{c}} P_{i}^{c}\left(\tau_{i}\right) d \tau_{i}-C_{i}\left(q_{i}^{c}\right)\right) \\
\text { subject to } & \left\{\begin{array}{l}
\sum_{i \in N} r_{i}^{c}=0 \\
\sum_{i \in N} D_{l, i}^{c} r_{i}^{c} \geq-K_{l}^{c}, l \in L \\
\sum_{i \in N} D_{l, i}^{c} r_{i}^{c} \leq K_{l}^{c}, l \in L
\end{array}\right.
\end{array}
$$

Here, $P_{i}^{c}(\cdot)$ and $C_{i}(\cdot)$ are the demand bid (or demand function) and the generation cost function at node $i$, respectively.

If we let $p^{c}, \lambda_{l-}^{c}$ and $\lambda_{l+}^{c}$ be the Lagrangian multipliers corresponding to the above constraints, the first order necessary conditions (the Karush-Kuhn-Tucker, KKT conditions) for the ISO's problem are a mixed linear complementarity problem (mixed LCP, see (Cottle, Pang and Stone 1992) ${ }^{[6]}$ ):

$$
\left\{\begin{array}{l}
P_{c}^{i}\left(q_{i}^{c}+r_{i}^{c}\right)-p^{c}+\sum_{m \in L}\left(\lambda_{m-}^{c} D_{m, i}^{c}-\lambda_{m+}^{c} D_{m, i}^{c}\right)=0, i \in N \\
\sum_{j \in N} r_{j}^{c}=0 \\
0 \leq \lambda_{l-}^{c} \perp \sum_{j \in N} D_{l, j}^{c} r_{j}^{c}+K_{l}^{c} \geq 0, l \in L \\
0 \leq \lambda_{l+}^{c} \perp K_{l}^{c}-\sum_{j \in N} D_{l, j}^{c} r_{j}^{c} \geq 0, l \in L[3 p t]
\end{array}\right.
$$

where the operator $\perp$ stands for the complementarity condition. The first KKT condition in (1) implies that nodal prices are of the form

$$
P_{c}^{i}\left(q_{i}^{c}+r_{i}^{c}\right)=p^{c}+\varphi_{i}^{c}
$$

where

$$
\varphi_{i}^{c}=-\sum_{m \in L}\left(\lambda_{m-}^{c} D_{m, i}^{c}-\lambda_{m+}^{c} D_{m, i}^{c}\right)
$$

Thus, the nodal demand function becomes

$$
q_{i}^{c}+r_{i}^{c}=\left(P_{c}^{i}\right)^{-1}\left(p^{c}+\varphi_{i}^{c}\right)
$$

and consequently, the aggregated system demand function is

$$
\sum_{j \in N} q_{j}^{c}=\sum_{j \in N}\left(P_{j}^{c}\right)^{-1}\left(p^{c}+\varphi_{j}^{c}\right)
$$

We can interpret $p^{c}$ as the energy price at the reference bus and $\left\{\varphi_{i}^{c}\right\}_{i \in N}$ as node specific premiums determining relative nodal prices. 


\subsubsection{The firms' decision making}

In the spot market, each firm $g \in G$ determines the outputs from its units. In our two-settlement model, we allow different granularity in the forward and spot markets. This is achieved by dividing the network into a set $Z$ of zones, each composed of a cluster of nodes. In particular, the spot market supply and demand at each node are settled at the so-called nodal prices, whereas the forward contracts are traded at the so-called zonal forward prices $\left\{h_{z}\right\}_{z \in Z}$ (see section 3.2), and settled at spot zonal settlement prices $\left\{u_{z}^{c}\right\}_{z \in Z}$ which are defined as weighted averages of the nodal prices in the zones, i.e.,

$$
u_{z}^{c}=\sum_{i: z(i)=z}\left(p^{c}+\varphi_{i}^{c}\right) \delta_{i}, \quad z \in Z
$$

where $z(i)$ and $\delta_{i}$ denote the zonal index and corresponding weight of node $i \in N$, respectively. In this paper, $\left\{\delta_{i}\right\}_{i \in N}$ are taken as historical load shares and not affected by market participants' decisions. In mathematical terms, each firm $g \in G$ solves the following profit-maximization problem that is parameterized by the forward prices $\left\{h_{z}\right\}_{z \in Z}$, the locational price premiums $\left\{\varphi_{i}^{c}\right\}_{i \in N}$ and its own forward contracts $\left\{x_{g z}\right\}_{z \in Z}$ :

$$
\begin{array}{ll}
\max _{\left\{q_{i}^{c}\right\}_{i \in N_{g}, p^{c}}} & \sum_{i \in N_{g}}\left(p^{c}+\varphi_{i}^{c}\right) q_{i}^{c}+\sum_{z \in Z}\left(h_{z}-u_{z}^{c}\right) x_{g, z}-\sum_{i \in N_{g}} C_{i}\left(q_{i}^{c}\right) \\
\text { subject to } & \left\{\begin{array}{l}
u_{z}^{c}=\sum_{i: z(i)=z}\left(p^{c}+\varphi_{i}^{c}\right) \delta_{i} \\
q_{i}^{c} \geq 0, i \in N_{g} \\
q_{i}^{c} \leq \bar{q}_{i}^{c}, i \in N_{g} \\
\sum_{j \in N} q_{j}^{c}=\sum_{j \in N}\left(P_{j}^{c}\right)^{-1}\left(p^{c}+\varphi_{j}^{c}\right)
\end{array}\right.
\end{array}
$$

Assume that the firms don't anticipate the impact of their productions on the locational price premiums, one can find the KKT conditions for firm $g$ 's program by letting $\rho_{i-}^{c}, \rho_{i+}^{c}$ and $\beta_{g}^{c}$ be the corresponding Lagrangian multipliers:

$$
\left\{\begin{array}{l}
p^{c}+\varphi_{i}^{c}-\beta_{g}^{c}-\frac{d C_{i}\left(q_{i}^{c}\right)}{d q_{i}^{c}}+\rho_{i-}^{c}-\rho_{i+}^{c}=0, \quad i \in N_{g} \\
\beta_{g}^{c} \sum_{j \in N} \frac{d\left(\left(P_{j}^{c}\right)^{-1}\right)\left(p^{c}+\varphi_{j}^{c}\right)}{d p^{c}}+\sum_{j \in N_{g}} q_{j}^{c}-\sum_{i \in N} \delta_{i} x_{g z(i)}=0 \\
\sum_{j \in N} q_{j}^{c}=\sum_{j \in N}\left(P_{j}^{c}\right)^{-1}\left(p^{c}+\varphi_{j}^{c}\right) \\
0 \leq \rho_{i-}^{c} \perp q_{i}^{c} \geq 0, \quad i \in N_{g} \\
0 \leq \rho_{i+}^{c} \perp \bar{q}_{i}^{c}-q_{i}^{c} \geq 0, \quad i \in N_{g}[3 p t]
\end{array}\right.
$$

Here, the first two conditions are the derivatives of the Lagrangian function with respect to $q_{i}^{c}$ and $p^{c}$, respectively.

Aggregating the KKT conditions for the firms' and the ISO's programs leads to the equilibrium conditions of the spot market as a mixed nonlinear complementarity problem. We assume that the demand side submits demand bids with fixed slope in different states, i.e., the demand bids are

$$
P_{i}^{c}(q)=a_{i}^{c}-b_{i} q, \quad i \in N, c \in C
$$

In addition, we let the cost functions be

$$
C_{i}(q)=d_{i} q+\frac{1}{2} s_{i} q^{2}, \quad i \in N
$$

then the spot-market equilibrium conditions become a mixed LCP 


$$
\left\{\begin{array}{l}
p^{c}+\varphi_{i}^{c}-\beta_{g}^{c}-d_{i}-s_{i} q_{i}^{c}+\rho_{i-}^{c}-\rho_{i+}^{c}=0, \quad i \in N_{g}, g \in G \\
p^{c}=a^{c}-\frac{\sum_{j \in N} \frac{\varphi_{j}}{b_{i}}}{\sum_{j \in N} \frac{1}{b_{i}}}-\frac{\sum_{j \in N} q_{j}^{c}}{\sum_{j \in N} \frac{1}{b_{i}}} \\
\varphi_{i}^{c}=-\sum_{m \in L}\left(\lambda_{m-}^{c} D_{m, i}^{c}-\lambda_{m+}^{c} D_{m, i}^{c}\right), \quad i \in N \\
-\beta_{g}^{c} \sum_{j \in N} \frac{1}{b_{i}}+\sum_{j \in N_{g}} q_{j}^{c}-\sum_{i \in N} \delta_{i} x_{g z(i)}=0, \quad g \in G \\
0 \leq \rho_{i-}^{c} \perp q_{i}^{c} \geq 0, \quad i \in N \\
0 \leq \rho_{i+}^{c} \perp \bar{q}_{i}^{c}-q_{i}^{c} \geq 0, \quad i \in N \\
\sum_{j \in N} r_{j}^{c}=0 \\
a^{c}-\left(q_{i}^{c}+r_{i}^{c}\right) b_{i}-p^{c}+\sum_{m \in L}\left(\lambda_{m-}^{c} D_{m, i}^{c}-\lambda_{m+}^{c} D_{m, i}^{c}\right), \quad i \in N \\
0 \leq \lambda_{l-}^{c} \perp \sum_{j \in N} D_{l, j}^{c} r_{j}^{c}+K_{l}^{c} \geq 0, l \in L \\
0 \leq \lambda_{l+}^{c} \perp K_{l}^{c}-\sum_{j \in N} D_{l, j}^{c} r_{j}^{c} \geq 0, l \in L
\end{array}\right.
$$

\subsection{The forward market}

In the forward market, the network feasibility constraints are ignored, and the forward contracts are traded at the forward prices (which in equilibrium will reflect expected network constraints in the spot market). We assume that the expected demand of the spot market appears in the forward market so that, for each node $i \in N$, its forward demand has the same slope as its spot demand function and the total area under the forward demand bid is equal to the expected area under the spot demand bid. This suggests that the nodal forward demand function is

$$
\begin{aligned}
P_{i}(q) & =E_{c}\left[a_{i}^{2}\right]-b_{i} q \\
& =\sum_{c \in C} \operatorname{Pr}(c)\left(a_{i}^{c}\right)^{2}-b_{i} q
\end{aligned}
$$

where $\operatorname{Pr}(c)$ denotes the probability of state $c$ in the spot market. This implies that the forward demand functions in the zones are

$$
P_{z}^{f w d}(q)=\frac{\sum_{i: z(i)=z} \frac{E_{c}\left[a_{i}^{2}\right]}{b_{i}}}{\sum_{i: z(i)=z} \frac{1}{b_{i}}}-\frac{q}{\sum_{i: z(i)=z} \frac{1}{b_{i}}}, \quad z \in Z
$$

and the forward market is cleared by the total forward contract at the forward prices:

$$
\begin{aligned}
h_{z} & =P_{z}^{f w d}\left(\sum_{g \in G} x_{g z}\right) \\
& =\frac{\sum_{i: z(i)=z} \frac{E_{c}\left[a_{i}^{2}\right]}{b_{i}}}{\sum_{i: z(i)=z} \frac{1}{b_{i}}}-\frac{\sum_{g \in G} x_{g z}}{\sum_{i: z(i)=z} \frac{1}{b_{i}}}, \quad z \in Z
\end{aligned}
$$

The rational firms simultaneously determine their forward contract quantities $\left\{x_{g z}\right\}_{g \in G, z \in Z}$ so as to maximize their expected utility, which is the expect profit if the firms are risk neutral, while anticipating the spot market outcome (3). Mathematically, each firm $g \in G$ solves the following mathematical program with equilibrium constraints (MPEC, see (Luo, Pang and Ralph 1996) ${ }^{[10]}$ ): 


$$
\begin{array}{ll}
\max _{\left\{x_{g, z}\right\} z Z} & \sum_{c \in C} \operatorname{Pr}(c) \pi_{g}^{c} \\
\operatorname{subject~to~} & \left\{\begin{array}{l}
\pi_{g}^{c}=\sum_{i \in N_{g}}\left(p^{c}+\varphi_{i}^{c}\right) q_{i}^{c}+\sum_{z \in Z}\left(h_{z}-u_{z}^{c}\right) x_{g, z}-\sum_{i \in N_{g}} C_{i}\left(q_{i}^{c}\right), \quad c \in C \\
h_{z}=\frac{\sum_{i: z(i)=z} \frac{E_{c}\left[a_{i}^{2}\right]}{b_{i}}}{\sum_{i: z(i)=z} \frac{1}{b_{i}}}-\frac{\sum_{g \in G} x_{g z}}{\sum_{i: z(i)=z} \frac{1}{b_{i}}}, \quad z \in Z \\
u_{z}^{c}=\sum_{i: z(i)=z}\left(p^{c}+\varphi_{i}^{c}\right) \delta_{i}, \quad z \in Z, c \in C \\
p^{c}+\varphi_{i}^{c}-\beta_{n}^{c}-d_{i}-s_{i} q_{i}^{c}+\rho_{i-}^{c}-\rho_{i+}^{c}=0, \quad i \in N_{g}, n \in G, c \in C \\
p^{c}=a^{c}-\frac{\sum_{j \in N} \frac{\varphi_{j}}{\sum_{i}}}{\sum_{j \in N} \frac{1}{b_{i}}}-\frac{\sum_{j \in N} q_{j}^{c}}{\sum_{j \in N} \frac{1}{b_{i}}}, c \in C \\
\varphi_{i}^{c}=-\sum_{m \in L}\left(\lambda_{m-}^{c} D_{m, i}^{c}-\lambda_{m+}^{c} D_{m, i}^{c}\right), \quad i \in N, c \in C \\
-\beta_{g}^{c} \sum_{j \in N} \frac{1}{b_{i}}+\sum_{j \in N_{g}} q_{j}^{c}-\sum_{i \in N} \delta_{i} x_{g z(i)}=0, g \in G, c \in C \\
0 \leq \rho_{i-}^{c} \perp q_{i}^{c} \geq 0, i \in N, c \in C \\
0 \leq \rho_{i+}^{c} \perp \bar{q}_{i}^{c}-q_{i}^{c} \geq 0, i \in N, c \in C \\
\sum_{j \in N} r_{j}^{c}=0 \\
a^{c}-\left(q_{i}^{c}+r_{i}^{c}\right) b_{i}-p^{c}+\sum_{m \in L}\left(\lambda_{m-}^{c} D_{m, i}^{c}-\lambda_{m+}^{c} D_{m, i}^{c}\right), \quad i \in N, c \in C \\
0 \leq \lambda_{l-}^{c} \perp \sum_{j \in N} D_{l, j}^{c} r_{j}^{c}+K_{l}^{c} \geq 0, l \in L, c \in C \\
0 \leq \lambda_{l+}^{c} \perp K_{l}^{c}-\sum_{j \in N} D_{l, j}^{c} r_{j}^{c} \geq 0, l \in L, c \in C
\end{array}\right.
\end{array}
$$

Hence, the overall problem in the markets is a so-called equilibrium problem with equilibrium constraints (EPEC). A solution to this problem is a set of the variables, including the firms' forward and spot quantities, the ISO's redispatch decisions and the corresponding Lagrangian multiplies, at which all firms' MPEC problems are simultaneously solved, and no market participant wants to unilaterally alter its decisions in either market.

\section{Agent-Based model}

One way to find the preceding equilibrium problem is to apply the solution approach described in (Yao, Adler and Oren 2005) ${ }^{[12]}$ for solving the MPEC problems (6). In this paper, we follow an agent-based model that simulates the market dynamics. In this framework, the market participants are modeled as autonomously acting software agents, who maximize their profits which they earn on electricity markets. Supply agents "learn" the profit maximizing quantity in both the spot and the forward market on the basis of a reinforcement learning algorithm.

The agent-based model is founded on a simple role framework. The dynamics of the spot and forward market evolves through the agents' determination of both the forward and the spot commitments, based on a best-of-practice approach. In every simulation step, results from last round's actions - if favorable - reinforce the tendency to choose this action again and weaken it in the opposite case. The simulation system is implemented under application of the Recursive Porous Agent Simulation Toolkit (RePast). RePast is a toolkit for modeling agent-based simulations. Many concepts available in RePast are based on Swarm, which has been developed at the Santa Fe Institute since 1994.

RePast allows to model large simulation systems based on software agents with individual characteristics. It incorporates an event scheduler, simulation control, data logging and visualization. In our model, we use RePast in order to include the role model introduced in the following section into an agent-based simulator.

\subsection{Roles and Model Description}

As shown in Fig. 1, the model comprises four different agent roles. Participants who request electricity are modeled as demand agents $\left\{D_{i}\right\}_{i \in N}$. The generation firms are conceived as supply agents $\left\{S_{i}\right\}_{i \in G}$. Supply agents own the power plants $\left\{G_{i}\right\}_{i \in N}$. Power transmission lines are handled by a transmission lines provider agent (TLP). Finally, the allocation between supply and demand is carried out by an independent system 
operator (ISO). The ISO is the central unit of the simulation system. It maintains a registry of all supplier and demand agents that is used to request bids from demand and supply agents in every round.

Both the forward and the spot market are carried out by the ISO. In the forward market, the forward price (5) is applied. In the spot market, the allocation between demand and supply is determined by solving the LCP problem (1). The outcomes of both markets are communicated back to the agents.

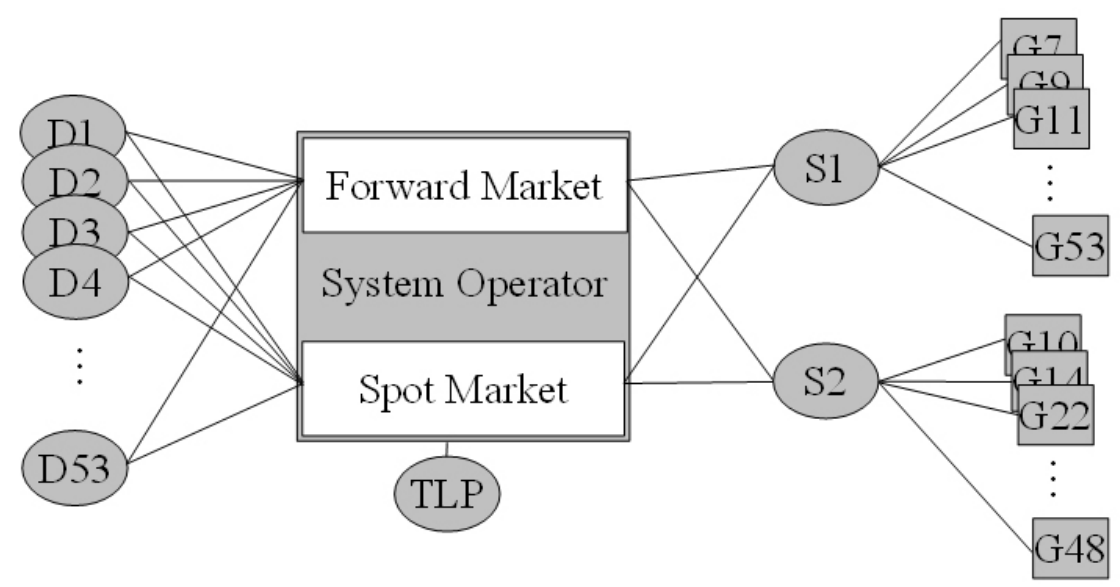

Fig. 1. Agent-based role model

\subsection{Reinforcement learning}

In this simulation model, only supply agents are modeled as adaptive agents. They implement a learning algorithm which is based on a modified version of the Erev-Roth reinforcement learning approach (Erev and Roth 1998) $)^{[8]}$ and (Nicolaisen, Petrov and Tesfatsion 2001) ${ }^{[11]}$.

The functionality of the Erev-Roth reinforcement learning algorithm is the following: For each situation in which learning is applied, an agent can chose from a set of possible actions $M$. In the given simulation scenario a supply agent chooses a bid quantity as a fraction of its available capacity. The continuous interval $[a, b]$ of possible fractions is stratified into $M=101$ discrete values, i.e. fractions from $[0,100]$ are possible actions.

If agent $n$ chooses his $k_{t h}$ action at time $t$ and receives a reinforcement of $R(x)$, it updates its individual propensities to choose action $j=1, \cdots, M$ according to the following inductive learning function:

$$
s_{n j}(t+1)=(1-\phi) \cdot s_{n j}(t)+\left\{\begin{array}{l}
R(x) \cdot(1-\varepsilon), \quad j=k \\
s_{n j}(t) \cdot \frac{\varepsilon}{M-1}, \quad \text { otherwise }
\end{array}\right.
$$

Here, $s_{n j}$ is the propensity to choose a specific action in the next round, $\phi$ is the "recency" parameter and $\varepsilon$ the "experimentation" parameter. The recency parameter reflects the agents' tendency to forget past experience over time, and the experimentation parameter has the effect that agents do not lock into one choice at a too early stage. Agents choose their action according to the following probability for action $k$ :

$$
p_{n k}(t+1)=\frac{s_{n k}(n+1)}{\sum_{j=1}^{M} s_{n j}(n+1)}
$$

The consequence of this formulation is that actions which have resulted in a high payoff are chosen again with a higher probability in the future. On the other hand, actions that lead to a lower payoff are chosen for another time with a lower probability. Agents are, thus, modeled as solving a myopic incentive-answer game. The results of all previous rounds are taken as a decision basis for the action to choose for the next round. 


\subsection{Simulation Interactions}

In our simulations the interactions between the agents are carried out in the following manner: In the forward market, the independent system operator sends a call for forward bids/offers to all demand and supply agents listed in his registry (this phase could represent a day-ahead market, for instance or a centralized forward market like Nordpool). Consequently, demand and supply agents compute and deliver their forward market bids/offers, represented by equation (4) and quantities $\left\{x_{g z}\right\}_{g \in G, z \in Z}$, respectively, to the ISO. The ISO then calculates the forward market allocation using a uniform price mechanism and communicates the resulting forward prices (5) to the demand and supply agents. In the spot market, demand and supply agents compute their spot market quantity bids/offers and communicate these to the ISO. Based on the submitted bids/offers, the ISO calculates the spot market allocation from the mixed LCP (1) and communicates the resulting spot prices (2) for the spot market to the demand and supply agents. Finally, demand and supply agents compute their profit and update their learned propensities based on both allocations. Subsequently, the next simulation step begins.

Agents learn spot bids/offers and forward bids/offers separately. They update their action propensities for spot market bids/offers on the basis of their achieved spot market profits. Learning is effected for each power plant and for each possible spot market state individually. The propensities for forward bid quantities are updated on the basis of the generator's total profit; quantities are chosen globally, i.e. learning is not differentiated for different plants.

\section{A Numerical Example}

\subsection{The Stylized Belgian Electricity Market}

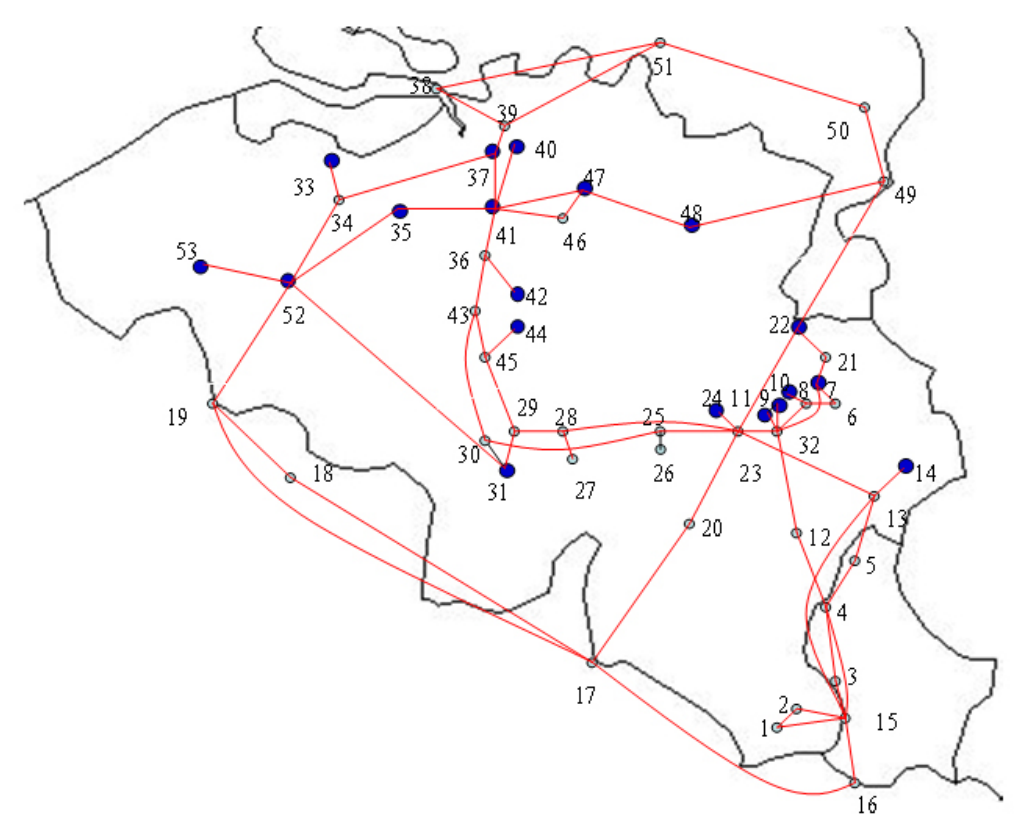

Fig. 2. The Belgian high voltage network

Since one of the main objectives of this paper is to validate the agent based approach against results obtained using computational equilibrium methods, we performed our simulation on a benchmark stylized version of the Belgian electricity system used in (Yao, Oren and Adler 2005b) ${ }^{[14]}$ and (Yao, Adler and Oren $2005)^{[12]}$. In this test case, the electrical network represents a realistic description of the Belgian system but the ownership structure, the nodal demand function the definition of zones and contingencies are hypothetical. The network has $92380 \mathrm{kV}$ and $220 \mathrm{kv}$ transmission lines, including certain lines in neighboring countries for 
their effect on loop flows. There are in total 53 buses (see Fig. 2), 19 of which are attached with generation plants. Insignificant lower voltage lines and small generation plants are excluded from this simulation.

Table 1. Generator information

\begin{tabular}{ccc|ccc}
\hline Node & $\begin{array}{c}\text { Marginal-cost } \\
(\$ / \mathrm{MWh})\end{array}$ & $\begin{array}{c}\text { Capacity } \\
(\mathrm{MW})\end{array}$ & Node & $\begin{array}{c}\text { Marginal-cost } \\
(\$ / \mathrm{MWh})\end{array}$ & $\begin{array}{c}\text { Capacity } \\
(\mathrm{MW})\end{array}$ \\
\hline 7 & 450 & 70 & 37 & 100 & 1399 \\
9 & 180 & 460 & 40 & 100 & 1378 \\
10 & 180 & 121 & 41 & 210 & 522 \\
11 & 200 & 124 & 42 & 180 & 385 \\
14 & 130 & 1164 & 44 & 200 & 538 \\
22 & 190 & 602 & 47 & 100 & 32 \\
24 & 100 & 2985 & 48 & 220 & 258 \\
31 & 180 & 712 & 52 & 580 & 95 \\
33 & 200 & 496 & 53 & 200 & 879 \\
35 & 250 & 1053 & & & \\
\hline
\end{tabular}

Table 2. Demand data

\begin{tabular}{ccc|ccc|ccc}
\hline Node & Slope & $\begin{array}{c}\text { Load } \\
\text { share }\end{array}$ & Node & Slope & $\begin{array}{c}\text { Load } \\
\text { share }\end{array}$ & Node & Slope & $\begin{array}{c}\text { Load } \\
\text { share }\end{array}$ \\
\hline 1 & 1.00 & 0.0292 & 19 & 0.68 & 0.0430 & 37 & 1.00 & 0.0424 \\
2 & 0.82 & 0.0356 & 20 & 1.05 & 0.0278 & 38 & 0.85 & 0.0499 \\
3 & 1.13 & 0.0258 & 21 & 1.00 & 0.0292 & 39 & 1.00 & 0.0424 \\
4 & 1.00 & 0.0292 & 22 & 1.10 & 0.0266 & 40 & 1.15 & 0.0369 \\
5 & 0.93 & 0.0314 & 23 & 1.00 & 0.0292 & 41 & 1.00 & 0.0424 \\
6 & 0.85 & 0.0344 & 24 & 0.75 & 0.0390 & 42 & 0.79 & 0.0537 \\
7 & 1.00 & 0.0292 & 25 & 1.00 & 0.0292 & 43 & 0.68 & 0.0624 \\
8 & 1.00 & 0.0292 & 26 & 0.80 & 0.0365 & 44 & 1.03 & 0.0412 \\
9 & 0.88 & 0.0332 & 27 & 1.13 & 0.0258 & 45 & 1.00 & 0.0424 \\
10 & 0.90 & 0.0325 & 28 & 1.00 & 0.0292 & 46 & 1.00 & 0.0424 \\
11 & 1.00 & 0.0292 & 29 & 0.93 & 0.0314 & 47 & 1.00 & 0.0424 \\
12 & 0.73 & 0.0400 & 30 & 0.85 & 0.0344 & 48 & 0.73 & 0.0581 \\
13 & 1.00 & 0.0292 & 31 & 1.00 & 0.0292 & 49 & 1.20 & 0.0354 \\
14 & 0.85 & 0.0344 & 32 & 1.00 & 0.0292 & 50 & 1.50 & 0.0283 \\
15 & 1.00 & 0.0292 & 33 & 0.88 & 0.0482 & 51 & 1.00 & 0.0424 \\
16 & 1.30 & 0.0225 & 34 & 0.50 & 0.0849 & 52 & 1.00 & 0.0424 \\
17 & 1.00 & 0.0292 & 35 & 1.00 & 0.0424 & 53 & 0.70 & 0.0606 \\
18 & 0.79 & 0.0370 & 36 & 0.73 & 0.0581 & & & \\
\hline
\end{tabular}

Tab. 1 lists the information of generation plants, including the capacities and marginal costs (assumed constant). In addition, we assume that there are two zones in the network with node 1 to 32 in the first zone and the remaining nodes in the second zone. Information on the slopes of the demand bids and the historical load shares can be found in Tab. 2. As to flow limits, we ignore the intra-zonal flows and focus only on the flowgates of lines 22-49, 29-45, 30-43 and 31-52 that connect the two zones.

We assume five contingency states in the spot market representing five possible realizations of demand, and system contingencies. In the first state, the demand levels are at the shoulder, all generation plants operate at their full capacities and all transmission lines are rated at their full thermal limits. The second state is the same as the first state except that it has on-peak demand. Off-peak state 3 differs from state 1 by the very low demand levels. State 4 and 5 capture the unavailability of two plants at node 10 and 41 , respectively. The assumed probabilities of these states are given in Tab. 3. For these states, the price intercepts for the nodal inverse demand functions are assumed uniform across the nodes and equal to 500, 1000, 250, 500 and 500, respectively. 
Table 3. States in the Belgian electricity network

\begin{tabular}{c|cl}
\hline State & Probability & Description \\
\hline 1 & 0.50 & Demand at shoulder \\
2 & 0.20 & Demand on peak \\
3 & 0.20 & Demand off peak \\
4 & 0.05 & Demand at shoulder; plant outage at node 10 \\
5 & 0.05 & Demand at shoulder; plant outage at node 41 \\
\hline
\end{tabular}

\subsection{Simulation results}

We run the simulation with two firms. The first firm owns the plants at the node set $\{7,9,11,31,32,33$, $35,37,41,47,53\}$, and the second firm at $\{10,14,22,24,40,42,44,48\}$. As stated early, only supply agents learn in our simulation scenario; demand agents submit bids with constant slopes. In the spot market, demand agents submit a price intercept of either 250,500, or 1000, depending on the states of the spot market, where as in the forward market, they submit demand functions at the price intercept of 602.08 .

100 simulation runs have been carried out for this stylized Belgian network. The parameter values have been chosen as $\phi=0.1$ and $\varepsilon=0.2$ and are thus equal to the values obtained by Erev and Roth (1998). They find that this combination fits best to their experimental results with human learning subjects. In our setup one simulation run comprises 1000 simulation steps. The first 900 steps are considered as the learning phase and the average values for prices and volumes of the last 100 simulation steps are evaluated. The evolution of a typical simulation run is shown in Figure 3. In the diagram Spot Market Price, the development of the spot market price over the time of 1000 simulation steps (days) can be seen. Diagram Spot Market Volume shows the corresponding spot market volume that is offered by the supply agents. In Forward Market Price, the development of the forward market price over the time of 1000 steps (days) is shown. The diagram Forward Market Volume shows the resulting volume in the forward market. The light gray diamonds represent the data in zone 1 whereas the dark dots refer to data in zone 2.

Tab. $4 \sim$ Tab. 6 summarize the producers' forward contracts, and the resulting forward and spot zonal prices, respectively. In both tables, we include the average, minimum, maximum and standard deviation obtained from the simulation. It is shown that both suppliers contract significant forward quantities. In addition, we observe that the forward prices are higher than the spot zonal prices; this is because, when facing the settlements for forward commitments, the suppliers attempt to raise forward prices and lower spot prices for cash inflows generated by forward contracts. This result is interesting since it deviates from the "no arbitrage constraint" imposed in the computational equilibrium approach (Yao, Adler and Oren 2005) ${ }^{[12]}$, equating forward prices to expected spot zonal prices.

Table 4. Forward contracting quantities (MW)

\begin{tabular}{l|cccc|cccc}
\hline & \multicolumn{4}{|c|}{ Zone 1 } & \multicolumn{4}{c}{ Zone 2 } \\
\cline { 2 - 9 } & Mean & Min & Max & SD $^{*}$ & Mean & Min & Max & SD $^{*}$ \\
\hline Firm 1 & 515.67 & 163.92 & 1078.37 & 246.02 & 1705.51 & 542.40 & 3568.46 & 812.44 \\
Firm 2 & 1342.11 & 48.72 & 4084.68 & 990.92 & 699.81 & 25.42 & 2133.28 & 517.63 \\
\hline
\end{tabular}

$*: \mathrm{SD}=$ standard deviation

Table 5. Forward contract prices ( $\$ / \mathrm{MWh})$

\begin{tabular}{l|cccc|cccc}
\hline & \multicolumn{4}{|c|}{ Zone 1 } & \multicolumn{4}{c}{ Zone 2 } \\
\cline { 2 - 9 } & Mean & Min & Max & SD $^{*}$ & Mean & Min & Max & SD $^{*}$ \\
\hline Forward prices & 547.82 & 461.08 & 591.88 & 29.92 & 499.98 & 403.11 & 568.27 & 41.10 \\
\hline *: SD = standard deviation
\end{tabular}



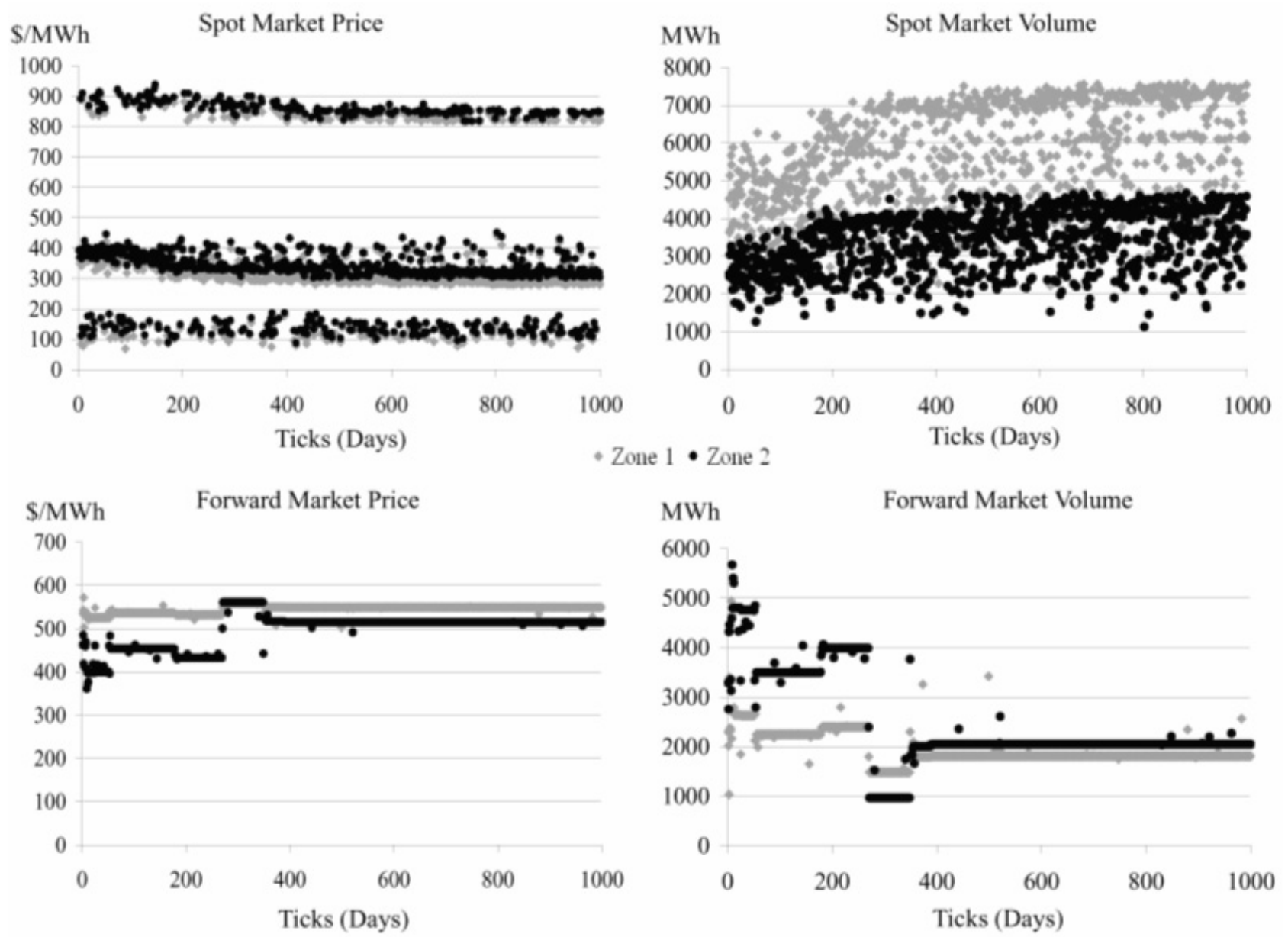

Fig. 3. Graphical output for one sample simulation of two settlements

Table 6. Spot zonal prices under two settlements(\$/MWh)

\begin{tabular}{c|cccc|cccc}
\hline & \multicolumn{5}{|c|}{ Zone 1 } & \multicolumn{4}{c}{ Zone 2 } \\
\cline { 2 - 9 } & Mean & Min & Max & SD & Mean & Min & Max & SD $^{*}$ \\
\hline State 1 & 319.73 & 286.83 & 385.26 & 18.70 & 341.80 & 312.61 & 390.76 & 16.22 \\
State 2 & 799.39 & 774.30 & 864.42 & 19.93 & 818.37 & 790.05 & 885.56 & 18.46 \\
State 3 & 127.06 & 111.10 & 149.20 & 8.04 & 141.65 & 126.51 & 159.94 & 7.18 \\
State 4 & 370.00 & 336.84 & 402.01 & 11.82 & 386.91 & 362.44 & 409.26 & 8.78 \\
State 5 & 374.45 & 342.74 & 414.58 & 12.48 & 396.09 & 368.87 & 432.99 & 11.28 \\
Average over states & 382.38 & - & - & - & 402.05 & - & - & - \\
\hline
\end{tabular}

$*: \mathrm{SD}=$ standard deviation

For comparison, we also simulate the outcomes of a single settlement where there is no forward market, (or equivalently, all firms in the two-settlement market can only submit zero forward contracts in the forward market). Fig. 4 illustrates a sample run of the simulation for a single settlement. The diagram Spot Market Price here represents the resulting spot market prices in case of a single settlement scenario. Diagram Spot Market Volume represents the volume allocated in these contracts. Here, also, the light diamonds represent the data in zone 1 whereas the dark dots show the data in zone 2.

We report in Tab. 7 the spot zonal prices in the single-settlement market. Comparing the prices under a single settlement to those under two settlements, we find that two settlements lead to lower spot market energy prices, and thus greater social welfare. Such difference is due to the increased spot market generation that results from the strategic effect of forward contracts. In other words, a forward market induces the suppliers to behave more competitively in the spot market. Tab. 8 compares the generation quantities under a single settlement to those under two settlements. As one would expect, the total generation in the markets is also 
higher with two settlements. Such results are qualitatively consistent with the conclusions in (Allaz 1992) ${ }^{[6]}$, (Kamat and Oren 2004) $)^{[9]}$ and (Yao, Adler and Oren 2005) ${ }^{[12]}$. Moreover, when comparing Tab. 6 to Tab. 7 , we find that the reduction of spot zonal prices in peak states is greater than that in off-peaks; this suggests that a forward market also yields less spot price volatility.

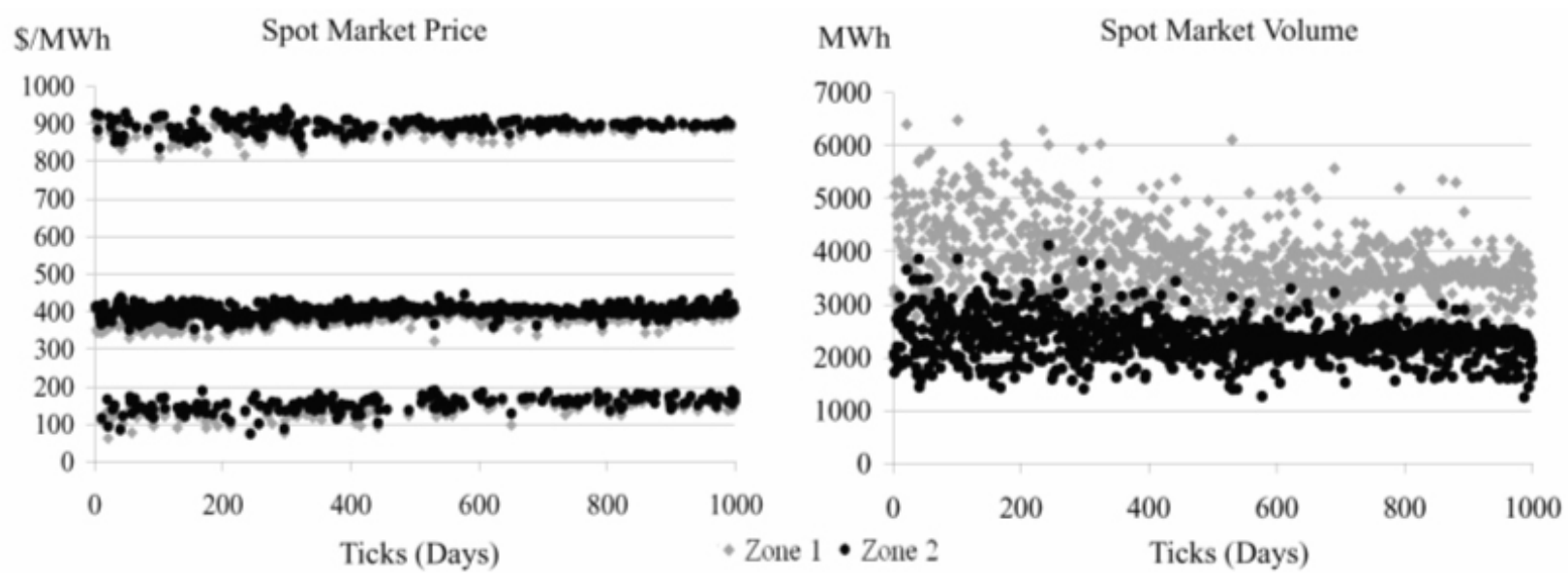

Fig. 4. Graphical output for a sample simulation of a single settlement

Table 7. Spot zonal prices under a single settlement $(\$ / \mathrm{MWh})$

\begin{tabular}{l|cccc|cccc}
\hline & \multicolumn{4}{|c|}{ Zone 1 } & \multicolumn{4}{c}{ Zone 2 } \\
\cline { 2 - 9 } & Mean & Min & Max & SD & Mean & Min & Max & SD $^{*}$ \\
\hline State 1 & 401.15 & 364.83 & 443.95 & 18.19 & 412.15 & 373.45 & 252.52 & 17.51 \\
State 2 & 884.34 & 838.90 & 934.27 & 20.55 & 889.47 & 861.70 & 939.20 & 17.00 \\
State 3 & 163.62 & 141.95 & 191.55 & 10.22 & 172.65 & 149.11 & 198.78 & 10.18 \\
State 4 & 379.78 & 346.88 & 428.39 & 14.25 & 396.82 & 354.34 & 438.00 & 12.51 \\
State 5 & 381.22 & 342.32 & 422.26 & 14.28 & 400.85 & 363.90 & 430.86 & 12.49 \\
\hline
\end{tabular}

Table 8. Spot supply (MW)

\begin{tabular}{c|c|cccc|cccc}
\hline \multirow{2}{*}{$\begin{array}{c}\text { Number of } \\
\text { settlements }\end{array}$} & \multirow{2}{*}{ State } & \multicolumn{5}{|c|}{ Zone 1 } & \multicolumn{4}{c}{ Zone 2 } \\
\cline { 3 - 10 } & & Mean & Min & Max & SD* & Mean & Min & Max & SD $^{*}$ \\
\hline \multirow{4}{*}{ Single } & 1 & 3382.92 & 1917.53 & 4626.48 & 622.99 & 2062.95 & 1111.28 & 2975.11 & 412.69 \\
& 2 & 3956.74 & 2247.32 & 5512.86 & 703.65 & 2377.30 & 1417.00 & 3244.00 & 400.77 \\
& 3 & 2956.78 & 2000.67 & 3698.74 & 349.84 & 1819.45 & 1203.33 & 2374.25 & 239.91 \\
& 4 & 4114.66 & 2450.16 & 5241.34 & 487.83 & 2424.17 & 1453.42 & 3425.82 & 294.80 \\
& 5 & 4065.45 & 2660.08 & 5397.35 & 489.06 & 2329.23 & 1621.85 & 3200.29 & 294.43 \\
\hline \multirow{4}{*}{ Two } & 1 & 6170.89 & 3927.01 & 7297.29 & 640.34 & 3720.77 & 2566.49 & 4409.06 & 382.33 \\
& 2 & 6865.68 & 4638.85 & 7724.51 & 682.56 & 4265.08 & 2680.88 & 4932.84 & 435.10 \\
& 3 & 4208.72 & 3450.63 & 4755.03 & 275.33 & 2549.64 & 2118.67 & 2906.67 & 169.23 \\
& 4 & 4449.74 & 3353.60 & 5585.02 & 404.66 & 2657.29 & 2130.50 & 3234.00 & 206.99 \\
& 5 & 4297.33 & 2923.14 & 5383.03 & 427.28 & 2440.91 & 1571.00 & 3082.50 & 266.07 \\
\hline
\end{tabular}

$*: \mathrm{SD}=$ standard deviation 


\section{Conclusions}

This paper studies the dynamics of a two-settlement electricity market that consists of a forward market and a spot market. Settlements for forward contracts and spot supplies are based on the zonal and nodal prices, respectively. In the forward market, suppliers submit strategic bids for forward contracts that are paid at the forward market clearing prices in the respective zones (or hubs). In the spot market, electricity generation is paid at the individual nodal prices and the forward contracts are settled at the spot zonal settlement prices.

We use an approach based on software agents to simulate the dynamics of such markets. Both the supply and demand sides are modeled as individual agents. On the supply side, every participating company is represented by one agent supporting zonal offers in the forward market and nodal offers for its generating units. The supply side agents are equipped with reinforcement learning algorithms which allow them to dynamically adapt their bidding strategies based on their success in the market. On the demand side, demand agents support inverse demand functions into the market as demand-side bids. The independent system operator (ISO) runs both the spot and the forward market, and carries out an allocation between the supply and the demand bids subject to transport network feasibility constraints.

Our simulations demonstrate that the introduction of a forward market creates incentives for suppliers to engage in forward contracts, which influence the supply agents' bidding strategies in the spot market. Moreover, such incentives result in lower energy prices and smaller price volatility as compared to a system without the forward market.

Extension of the research will include the sensitivity analysis of supplies' forward commitments and spot behaviors on market concentration. We also plan to include risk aversion in suppliers' utility functions. The impact of regulatory price/bid caps in the spot market is also a subject of future work.

\section{Acknowledgments}

This research is partially supported by the US National Science Foundation Grant ECS-0224779, by the Power System Engineering Research Center (PSERC), by the University of California Energy Institute (UCEI), by the Consortium for Electric Reliability Technology Solutions (CERTS) the US Department of Energy and by Volkswagen Foundation under Grant No. II/79625.

\section{References}

[1] B. Allaz. Oligopoly, Uncertainty and Strategic Forward Transactions. Internal Journal of Industrial Organization, 1992, 10: 297-308.

[2] B. Allaz, J. L. Vila. Cournot Competition, Forward Markets and Efficiency. Journal of Economic Theory, 1993, 59: $1-16$.

[3] J. Bower, D. W. Bunn. Model-Based Comparison of Pool and Bilateral Markets for Electricity. The Energy Journal, 2000, 21(3): 1-29.

[4] J. Bower, D. W. Bunn. Experimental Analysis of the Efficiency of Uniform-price for Electricity. Journal of Economics Dynamics Control, 2001, 25: 561-592.

[5] H. P. Chao, S. C. Peck. A market mechanism for electric power transmission. Journal of Regulatory Economics, 1996, 10(1): 25-60.

[6] R. W. Cottle, J. S. Pang, R. E. Stone. The Linear Complementarity Problem. Academic Press, Boston, 1992.

[7] C. Czernohous, W. Fichtner, et al. Management Decision Support using Long-T]erm Market Simulation, volume $=1$, year $=2003$. Journal of Information Systems and e-Business Management (ISeB), (4): 405-423.

[8] I. Erev, A. Roth. Predicting How People Play Games: Reinforcement Learning in Experimental Games with Unique, Mixed Strategy Equilibria. American Economic Review, 1998, 88(4): 848-881.

[9] R. Kamat, S. S. Oren. Multi-Settlement Systems for Electricity Markets: Zonal Aggregation under Network Uncertainty and Market Power. Journal of Regulatory Economics, 2004, 25(1): 5-37.

[10] Z. Q. Luo, J. S. Pang, D. Ralph. Mathematical Programs with Equilibrium Constraints. Cambridge University press, Cambridge, 1996.

[11] J. Nicolaisen, V. Petrov, L. Tesfatsion. Market Power and Efficiency in a Computational Electricity Market With discriminatory Double-Auction Pricing. IEEE Transactions on Evolutionary Computation, 2001, 5(5): 504-523. 
[12] J. Yao, I. Adler, S. S. Oren. Modeling and Computing Two-settlement Oligopolistic Equilibrium in a Congested Electricity Network. California, 2005. Manuscript.

[13] J. Yao, S. S. Oren, I. Adler. Cournot Equilibria in Two-Settlement Electricity Markets with System Contingencies. 2005. Forthcoming.

[14] J. Yao, S. S. Oren, I. Adler. Two-Settlement Electricity Markets with Price Caps and Cournot Generation Firms. 2005. In Press. 